\title{
Indirect Adaptive Attitude Control for a Ducted Fan Vertical Takeoff and Landing Microaerial Vehicle
}

\author{
Shouzhao Sheng, ${ }^{1}$ Chenwu Sun, ${ }^{1}$ and Hong Zhao ${ }^{2}$ \\ ${ }^{1}$ College of Automation Engineering, Nanjing University of Aeronautics and Astronautics, 29 YuDao Street, Nanjing 210016, China \\ ${ }^{2}$ Laboratory of Science and Technology on Rotorcraft Aeromechanics, Nanjing University of Aeronautics and Astronautics, \\ 29 YuDao Street, Nanjing 210016, China
}

Correspondence should be addressed to Shouzhao Sheng; shengsz@nuaa.edu.cn

Received 22 March 2015; Revised 15 June 2015; Accepted 15 June 2015

Academic Editor: Victor Sreeram

Copyright (C) 2015 Shouzhao Sheng et al. This is an open access article distributed under the Creative Commons Attribution License, which permits unrestricted use, distribution, and reproduction in any medium, provided the original work is properly cited.

\begin{abstract}
The present paper addresses an attitude tracking control problem of a ducted fan microaerial vehicle. The proposed indirect adaptive controller can greatly reduce tracking error in the initial stage of the adaptive learning process by using an error compensation strategy and can achieve good capability to eliminate the adverse effect of measurement noises on the convergence of adjustable parameters. Moreover, the learning rate adaptation strategy is proposed to further minimize the adverse effect of large learning rates on the convergence of adjustable parameters. The experimental tests have illustrated the effectiveness of the proposed adaptive controller.
\end{abstract}

\section{Introduction}

Ducted fan microaerial vehicles (MAVs) are a kind of unmanned aerial vehicles with small sizes and compact structures, which are usually capable of low-speed flight in addition to the normal hover and vertical takeoff and landing capabilities. With both military and civilian applications, they have a great technological potential in arduous or hazardous mission like surveillance, inspection, exploration, intelligence reconnaissance, target acquisition, and signal relay [1]. Compared to a conventional configuration microunmanned helicopter (MUH) or a multirotor craft (MRC), the ducted fan MAV is a much more safe platform since its propeller is mounted inside the duct which can act as a shield that helps avoid the risk of injury. MUHs and MRCs, however, are rather dangerous due to the exposed rotor blades.

Ducted fan aerodynamics has been widely explored on the piloted aerial vehicle platforms in the mid-20th century [2-4]. For a long time, these projects were almost under stagnation because these ducted fan manned vehicles appeared to be inferior to conventional rotorcrafts in cost-effectiveness. However, with the increasing research enthusiasm on unmanned systems over the past decades, ducted fan VTOL MAVs gradually attracted great interests.
As a consequence, a series of such prototype MAVs have achieved stable flight, such as Sikorsky Cypher [5, 6], Allied Aerospace iSTAR9 [7], and Bertin Technologies HoverEye [8].

Great effort has been made for ducted fan MAVs, including optimal design $[9,10]$, aerodynamic modeling, system identification [11-13], and flight control design [1417]. As for the attitude control problem of ducted fan MAVs, PID controller [18] is the most popular choice, but it heavily relies on real-time parameter adjustment according to flight conditions and therefore cannot cover the full flight envelope. A sliding mode technique is developed in [19], which shows superior performance over classical loopshaping techniques when variations in vehicle dynamics and actuator characteristics are introduced; however, the resulting performance would be deteriorated in practical applications because of measurement noises. A backstepping method was applied to HoverEye in [8], which can overcome the problem of gyroscopic coupling, whereas the effect of measurement noises on the resulting performance has not been systematically addressed. Spaulding et al. [20] adopted a nonlinear dynamic inversion method, which is well suited for mission and failure reconfiguration; however, it demands an accurate flight dynamic model and full state feedback. 
In practice, it is very difficult to obtain accurate models because of parametric uncertainties. The image-based visual feedback scheme is designed to stabilize the system in a large domain combined with adaptive filtering strategy in [21], which, however, achieved satisfactory performance on the basis of high performance camera and image processing capability.

Other existing methods include linear-quadratic regulator (LQR) and particle swarm optimization (PSO) [22], robust control method [23], neural adaptive method [24], and fuzzy techniques $[25,26]$. Although such of the aforementioned control methods are designed for ducted fan MAVs, little attention has been paid to dealing with parametric uncertainties and measurement noises. In [27], a modified model reference adaptive control strategy considers the adverse effect of measurement noises, but it merely reduces the effect by using a combination of low- and high-pass filters and could not guarantee the convergence of adjustable parameters of the test plant.

This paper focuses on the attitude tracking control problem of a ducted fan MAV. An indirect adaptive control scheme is proposed in the presence of parametric uncertainties and measurement noises. The proposed adaptive control scheme is able to effectively reduce the tracking error in the initial stage of the adaptive learning process and eliminate the adverse effect of measurement noises on the convergence of adjustable parameters. Moreover, a learning rate adaptation strategy is proposed to further minimize the adverse effect of large learning rates on the convergence of adjustable parameters.

This paper is organized as follows. Section 2 is devoted to the description of a small-size ducted fan VTOL MAV and its attitude control problem. The indirect adaptive control scheme is formulated in Section 3 and experimental tests are presented in Section 4. The last section offers conclusions.

\section{Ducted Fan MAV Description and the Attitude Control Problem Statement}

This study is based on a small-size ducted fan VTOL MAV model shown in Figure 1. This vehicle is propelled by a ducted fan. The attitude control is achieved by adjusting control vanes at the tail of the duct. Most of antitorque generated by the ducted fan is compensated by stators configured inside the duct while the remaining antitorque is balanced by controlling vanes.

For this ducted fan MAV, six-degree-of-freedom (6-DOF) nonlinear kinematic equations can be defined as follows:

$$
\dot{\widehat{\mathbf{x}}}=f(\widehat{\mathbf{x}}, \widehat{\mathbf{u}}, \Theta),
$$

where $\widehat{\mathbf{x}}=[\widehat{u}, \widehat{v}, \widehat{w}, \widehat{\theta}, \widehat{\phi}, \widehat{\psi}, \widehat{q}, \widehat{p}, \widehat{r}]^{\mathrm{T}}$ and $\widehat{\mathbf{u}}=\left[\widehat{\delta}_{p}, \widehat{\delta}_{r}, \widehat{\delta}_{y}, \widehat{\delta}_{t}\right]^{\mathrm{T}}$ denote the state vector and the system input vector, respectively, and $\Theta$ represents the aerodynamic parameter set, which is unavailable in most cases.

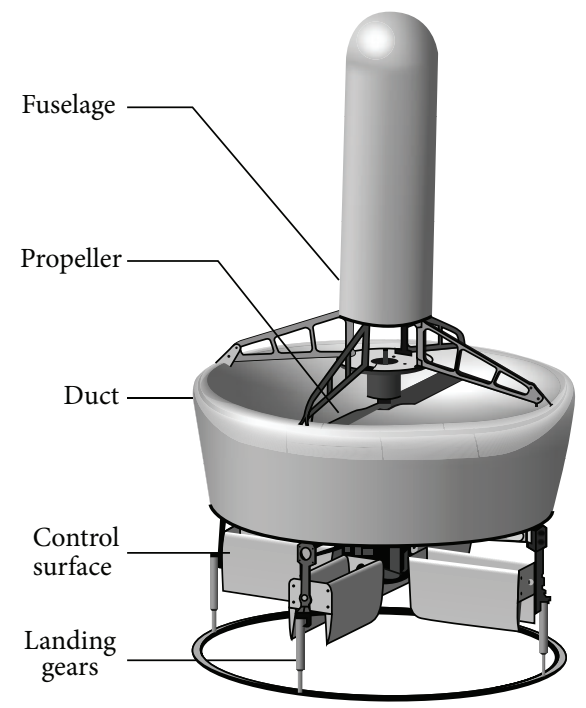

Figure 1: Ducted fan VTOL MAV.

The nonlinear system described by (1) has been linearized and the linearized model is given by

$$
\dot{\mathbf{x}}=\mathbf{A x}+\mathbf{B u}
$$

where $\mathbf{x}=\widehat{\mathbf{x}}-\mathbf{x}_{e}=[u, v, w, \theta, \phi, \psi, q, p, r]^{\mathrm{T}}$ and $\mathbf{u}=\widehat{\mathbf{u}}-$ $\mathbf{u}_{e}=\left[\delta_{p}, \delta_{r}, \delta_{y}, \delta_{t}\right]^{\mathrm{T}}$. Accordingly, $\mathbf{x}_{e}$ and $\mathbf{u}_{e}$ should satisfy the equation:

$$
f\left(\mathbf{x}_{e}, \mathbf{u}_{e}, \Theta\right)=0 \text {. }
$$

The determination of $\mathbf{x}_{e}$ and $\mathbf{u}_{e}$ is more complex in the presence of the parametric uncertainties in $\Theta$. Therefore, we define

$$
\begin{aligned}
& \mathbf{x}_{0}=\mathbf{x}_{e}+\mathbf{e}_{x} \\
& \mathbf{u}_{0}=\mathbf{u}_{e}+\mathbf{e}_{u}
\end{aligned}
$$

which are the estimates of $\mathbf{x}_{e}$ and $\mathbf{u}_{e}$, respectively. Equation (2) can then be rewritten as

$$
\dot{\mathbf{x}}=\mathbf{A x}+\mathbf{B u}+\mathbf{e},
$$

where $\mathbf{x}=\widehat{\mathbf{x}}-\mathbf{x}_{0}, \mathbf{u}=\widehat{\mathbf{u}}-\mathbf{u}_{e}$ and $\mathbf{e}=\mathbf{A} \mathbf{e}_{x}+\mathbf{B} \mathbf{e}_{u}$.

Apparently, the complexity of the control design mainly comes from the fact that $\mathbf{A}, \mathbf{B}, \mathbf{e}$ are unknown under most of flight conditions.

The goal of this study is to design a reliable and robust attitude controller for the ducted fan MAV in the presence of parametric uncertainties and measurement noises.

\section{Design of the Indirect Adaptive Controller}

3.1. Schematic Block Diagram. In general, the ducted fan MAV can be roughly regarded as a four-axis vehicle, that 


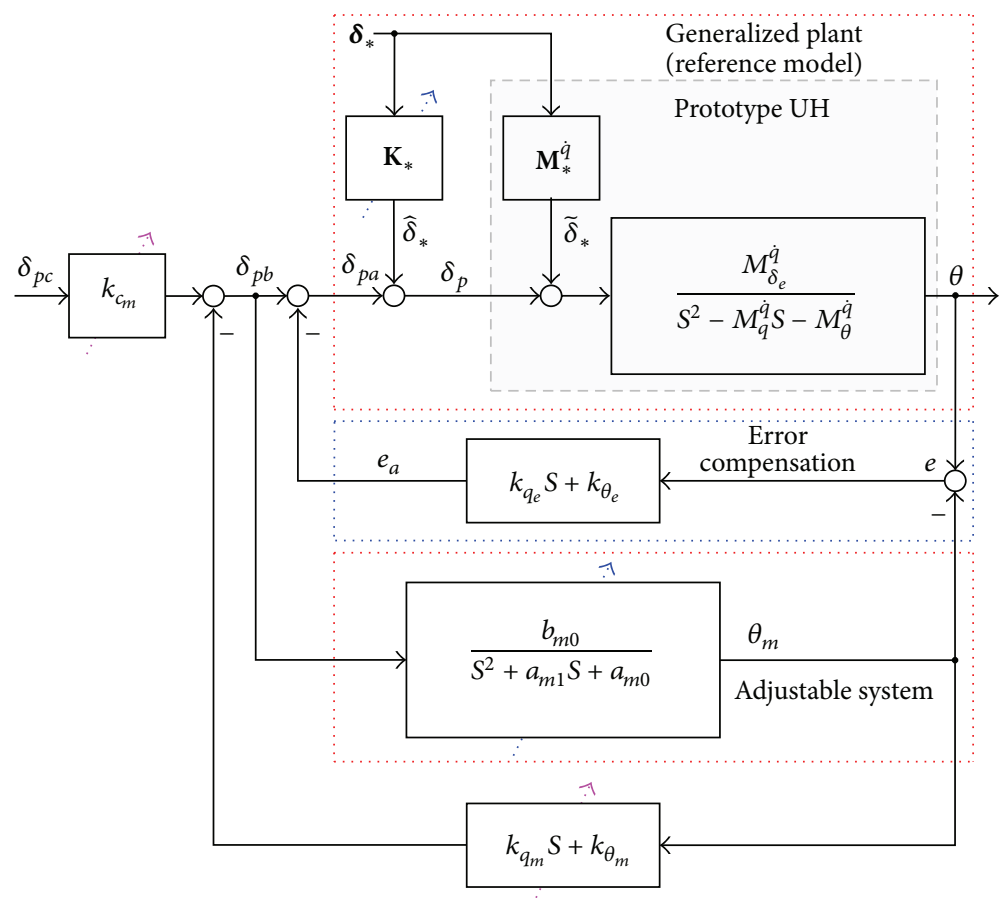

FIgURE 2: Control system block diagram.

is, vertical, longitudinal, lateral, and directional axes. Each axis can then be controlled independently with interactions with others. In particular, the longitudinal and lateral axes have the same properties because the MAV is central symmetrical. To avoid repetition, the present study is focused on the longitudinal axis in the hovering flight condition to demonstrate the proposed control scheme, which, without loss of generality, can apply to other axes. Thus, referring to (5), the linearized model of the longitudinal axis is given by

$$
\begin{aligned}
\dot{q}= & M_{u}^{\dot{q}} u+M_{v}^{\dot{q}} v+M_{w}^{\dot{q}} w+M_{\theta}^{\dot{q}} \theta+M_{\phi}^{\dot{q}} \phi+M_{\psi}^{\dot{q}} \psi \\
& +M_{q}^{\dot{q}} q+M_{p}^{\dot{q}} p+M_{r}^{\dot{q}} r+M_{\delta_{p}}^{\dot{q}} \delta_{p}+M_{\delta_{r}}^{\dot{q}} \delta_{r}+M_{\delta_{y}}^{\dot{q}} \delta_{y} \\
& +M_{\delta_{t}}^{\dot{q}} \delta_{t}+\eta,
\end{aligned}
$$

where $M_{\bullet}^{\dot{q}} \in \Theta$ and $\eta$ is added to represent the unknown trim error.

With respect to the ducted fan MAV, there exist $M_{v}^{\dot{q}}, M_{\phi}^{\dot{q}}, M_{\psi}^{\dot{q}}, M_{r}^{\dot{q}}, M_{\delta_{y}}^{\dot{q}} \approx 0$ and $q \approx \dot{\theta}$. Thus, (6) can be simplified as

$$
\ddot{\theta}-M_{q}^{\dot{q}} \dot{\theta}-M_{\theta}^{\dot{q}} \theta=M_{\delta_{p}}^{\dot{q}}\left(\delta_{p}+\widetilde{\delta}_{*}\right) .
$$

Here $\widetilde{\delta}_{*}$ is viewed as an external disturbance and can be expressed as

$$
\tilde{\delta}_{*}=\mathbf{M}_{*}^{\dot{q}} \boldsymbol{\delta}_{*}
$$

where

$$
\begin{aligned}
\mathbf{M}_{*}^{\dot{q}} & =\left[\frac{M_{u}^{\dot{q}}}{M_{\delta_{p}}^{\dot{q}}}, \frac{M_{w}^{\dot{q}}}{M_{\delta_{p}}^{\dot{q}}}, \frac{M_{p}^{\dot{q}}}{M_{\delta_{p}}^{\dot{q}}}, \frac{M_{\delta_{r}}^{\dot{q}}}{M_{\delta_{p}}^{\dot{q}}}, \frac{M_{\delta_{t}}^{\dot{q}}}{M_{\delta_{p}}^{\dot{q}}}, \frac{\eta}{M_{\delta_{p}}^{\dot{q}}}\right], \\
\boldsymbol{\delta}_{*} & =\left[u, w, p, \delta_{r}, \delta_{t}, 1\right]^{\mathrm{T}} .
\end{aligned}
$$

The schematic block diagram of the indirect adaptive attitude tracking controller is constructed, as shown in Figure 2, which includes a generalized plant, an adjustable system, and an error compensation module.

Unlike most existing adaptive schemes, the mathematical model is regarded as the adjustable system substituting for the generalized plant in the abovementioned adaptive control scheme. The purpose is to eliminate the adverse effect of measurement noises on the convergence of adjustable parameters, which will be discussed in detail in the following sections. The error compensation module is introduced to reduce the tracking error especially in the initial stage of the adaptive learning process, but, on the other hand, this module also decreases adaptive learning rates because of the insignificant tracking error. $a_{m_{0}}, a_{m_{1}}, b_{m_{0}}$, the parameters of the mathematical model, are therefore chosen as a part of adjustable parameters, which together with $\mathbf{K}_{*}$ are determined using an adaptive regulation strategy. $k_{c_{m}}$, $k_{q_{m}}, k_{\theta_{m}}$, another part of adjustable parameters, can be determined using an indirect adaptive pole placement control algorithm on the basis of the known parameters of the mathematical model. Note that the adjustable parameter vector $\mathbf{K}_{*}$ is used to remove the cross coupling among the axes. 
3.2. Design of Indirect Adaptive Control Strategy. The generalized plant and the adjustable system model are given as follows:

$$
\begin{aligned}
\ddot{\theta}+a_{1} \dot{\theta}+a_{0} \theta & =b_{0} \delta_{e a}+\Delta \mathbf{B}_{*} \boldsymbol{\delta}_{*} \\
\ddot{\theta}_{m}+a_{m_{1}} \dot{\theta}+a_{m_{0}} \theta_{m} & =b_{m_{0}} \delta_{p b},
\end{aligned}
$$

where $a_{0}=-M_{\theta}^{\dot{q}}, a_{1}=-M_{q}^{\dot{q}}, b_{0}=M_{\delta_{p}}^{\dot{q}}$, and $\Delta \mathbf{B}_{*}=\left(\mathbf{K}_{*}+\right.$ $\left.\mathbf{M}_{*}^{\dot{q}}\right) M_{\delta_{p}}^{\dot{q}}$.

From Figure 2, the tracking error is given by

$$
e=\theta-\theta_{m}
$$

which can be derived as

$$
\begin{aligned}
\ddot{e}+\widehat{a}_{1} \dot{e}+\widehat{a}_{0} e= & -\Delta a_{m_{1}} \dot{\theta}_{m}-\Delta a_{m_{0}} \theta_{m}+\Delta b_{m_{0}} \delta_{p b} \\
& +\Delta \mathbf{B}_{*} \boldsymbol{\delta}_{*},
\end{aligned}
$$

where $\widehat{a}_{0}=a_{0}+b_{0} k_{\theta_{e}}, \widehat{a}_{1}=a_{1}+b_{0} k_{q_{e}}, \Delta a_{m_{1}}=a_{1}-a_{m_{1}}, \Delta a_{m_{0}}=$ $a_{0}-a_{m_{0}}$, and $\Delta b_{m_{0}}=b_{0}-b_{m_{0}}$. Equation (12) indicates that the system stability can be guaranteed based on the proper choice of $k_{\theta_{e}}, k_{q_{e}}$ and the a priori knowledge of the ducted fan MAV parameters.

The system described by (12) is therefore hyperstable if the forward path transfer function is strictly positive real, and the input and output of the nonlinear feedback block satisfy Popov's integral inequality [28].

For this purpose, the indirect adaptive control laws are specified by

$$
\begin{aligned}
& a_{m_{0}}=-\sigma_{a_{m_{0}}} \varepsilon \theta_{m}, \\
& a_{m_{1}}=-\sigma_{a_{m_{1}}} \varepsilon \theta_{m}, \\
& b_{m_{0}}=-\sigma_{b_{m_{0}}} \varepsilon \delta_{p b}, \\
& \dot{\mathbf{K}}_{*}=-\boldsymbol{\sigma}_{*} \varepsilon \boldsymbol{\delta}_{*},
\end{aligned}
$$

where $\sigma_{a_{m_{0}}}, \sigma_{a_{m_{1}}}, \sigma_{b_{m_{0}}}$, and $\sigma_{*}=\operatorname{diag}\left(\sigma_{u}, \sigma_{w}, \sigma_{p}, \sigma_{\delta_{r}}, \sigma_{\delta_{t}}, \sigma_{\eta}\right)$ are learning coefficients; and

$$
\varepsilon=\tau_{q}\left(\dot{\theta}-\dot{\theta}_{m}\right)+\tau_{\theta}\left(\theta-\theta_{m}\right)
$$

where $\tau_{q}, \tau_{\theta}$ should be properly chosen such that $\left(\tau_{q} s+\right.$ $\left.\tau_{\theta}\right) /\left(s^{2}+\widehat{a}_{1} s+\widehat{a}_{0}\right)$ is strictly positive real. Therefore,

$$
\frac{\tau_{q}}{\tau_{\theta}}>\frac{1}{\widehat{a}_{1}} \quad \tau_{\theta}>0 .
$$

The adaptive control laws given by (13) can also be regarded as an adaptive identification solution with dynamic decoupling.
According to the Aeronautical Design Standard (ADS-33) Performance Specification, we assume that the ideal model is specified by the input-output relation

$$
\frac{\theta_{m}(s)}{\delta_{e c}(s)}=\frac{b_{m_{0}}^{*}}{s^{2}+a_{m_{1}}^{*} s+a_{m_{0}}^{*}}
$$

where $b_{m_{0}}^{*}, a_{m_{1}}^{*}, a_{m_{0}}^{*}$ are determined to meet the handling qualities and stability margin requirements of ADS-33. The adjustable parameters shown in Figure 2 are then updated by

$$
\begin{aligned}
& k_{c_{m}}=\frac{b_{m_{0}}^{*}}{b_{m_{0}}}, \\
& k_{q_{m}}=\frac{a_{m_{1}}^{*}-a_{m_{1}}}{b_{m_{0}}}, \\
& k_{\theta_{m}}=\frac{a_{m_{0}}^{*}-a_{m_{0}}}{b_{m_{0}}} .
\end{aligned}
$$

The output of the generalized plant can therefore track that of the ideal model based on the above adaptive strategies given by (13) and (17).

\subsection{Unbiased Analysis of Adjustable Parameters. Denote}

$$
\begin{aligned}
& \theta=\theta_{0}+\xi_{\theta}, \\
& \dot{\theta}=\dot{\theta}_{0}+\xi_{q},
\end{aligned}
$$

where $\xi_{\theta}, \xi_{q}$ have zero means and are uncorrelated with each other. From (14) and (18), we have

$$
\varepsilon=\varepsilon_{0}+\xi_{\varepsilon}
$$

where

$$
\begin{aligned}
& \varepsilon_{0}=\tau_{q}\left(\dot{\theta}_{0}-\dot{\theta}_{m}\right)+\tau_{\theta}\left(\theta_{0}-\theta_{m}\right), \\
& \xi_{\varepsilon}=\tau_{\theta} \xi_{\theta}+\tau_{q} \xi_{q} .
\end{aligned}
$$

Thus,

$$
\begin{aligned}
& E\left\{a_{m_{0}}(t)\right\}=a_{m_{0}}(0)-\sigma_{a_{m_{0}}} \int_{0}^{t} \varepsilon_{0}(\tau) \theta_{m}(\tau) d \tau, \\
& E\left\{a_{m_{1}}(t)\right\}=a_{m_{1}}(0)-\sigma_{a_{m_{1}}} \int_{0}^{t} \varepsilon_{0}(\tau) \dot{\theta}_{m}(\tau) d \tau, \\
& E\left\{b_{m_{0}}(t)\right\}=b_{m_{0}}(0)-\sigma_{b_{m_{0}}} \int_{0}^{t} \varepsilon_{0}(\tau) \delta_{p b}(\tau) d \tau, \\
& E\left\{\mathbf{K}_{*}(t)\right\}=\mathbf{K}_{*}(0)-\boldsymbol{\sigma}_{*} \int_{0}^{t} \varepsilon_{0}(\tau) \boldsymbol{\delta}_{*}(\tau) d \tau,
\end{aligned}
$$

which indicate that the adaptive parameters determined by (13) are therefore unbiased. 
However, if the generalized plant is regarded as an adjustable system substituting for the mathematical model in Figure 2, then the adaptive laws include terms like $\varepsilon \cdot \theta$ and $\varepsilon \cdot \dot{\theta}$ as many existing cases (e.g., [27]). This means that, from (18), (19), and (20), adjustable parameters cannot converge to the desired values due to measurement noises.

3.4. Design of Error Compensation. Although the indirect adaptive control strategy has been employed, the tracking error $e$ is nonnegligible during the adaptive learning process, especially in the initial stage. Referring to (12), the tracking error can be rewritten as

$e(s)$

$$
=\frac{-\widetilde{a}_{m_{0}} \theta_{m}(s)-\widetilde{a}_{m_{1}} \dot{\theta}_{m}(s)+\widetilde{b}_{m_{0}} \delta_{p b}(s)+\widetilde{\mathbf{B}}_{*} \boldsymbol{\delta}_{*}(s)}{\widehat{D}(s)},
$$

where $\tilde{a}_{m_{0}}=\Delta a_{m_{0}} / \widehat{a}_{0}, \tilde{a}_{m_{1}}=\Delta a_{m_{1}} / \widehat{a}_{0}, \widetilde{b}_{m_{0}}=\Delta b_{m_{0}} / \widehat{a}_{0}$, $\widetilde{\mathbf{B}}_{*}=\Delta \mathbf{B}_{*} / \widehat{a}_{0}$, and $\widehat{D}(s)=\left(1 / \widehat{a}_{0}\right) s^{2}+\left(\widehat{a}_{1} / \widehat{a}_{0}\right) s+1$. Thus, the tracking error $e$ can be reduced by choosing $k_{\theta_{e}}, k_{q_{e}}$ and adaptive learning.

Based on the a priori knowledge of the ducted fan MAV parameters, the proper choice of $k_{\theta_{e}}, k_{q_{e}}$ can therefore guarantee the stability of the generalized plant and reduce the tracking error during the adaptive learning process, especially in the initial stage. The tracking error can be limited to a given bounded range during the whole adaptive learning process while $\delta_{p c}$ and $\boldsymbol{\delta}_{*}$ remain bounded at all times. $e$ is gradually eliminated as $\Delta a_{m_{0}}, \Delta a_{m_{1}}, \Delta b_{m_{0}}, \Delta \mathbf{B}_{*} \rightarrow 0$ in the later stage of adaptive learning process.

3.5. Improvement of the Adaptive Laws. Note that in (13) the learning rates are related to $\varepsilon, \delta_{p b}, \theta_{m}, \dot{\theta}_{m}$, and $\boldsymbol{\delta}_{*}$. In fact, $\theta_{m}$, $\dot{\theta}_{m}$ are determined by $\delta_{p c}$, and $\delta_{e b}$ is determined not only by $\delta_{p c}$, but also by $k_{c_{m}}, k_{q_{m}}, k_{\theta_{m}}$. Large learning rates can easily cause the parametric oscillation in the adjustable system, which negatively affect the convergence rates of adjustable parameters.

To avoid this problem, the adaptive laws need to be improved through learning rate adaptation. The learning coefficient $\sigma_{a_{m_{0}}}$ is then replaced by

$$
\sigma_{a_{m_{0}}}=\sigma_{a_{m_{0}}}^{*} \exp \left(-\lambda_{a_{m_{0}}}\left|\varepsilon \theta_{m}\right|\right)
$$

where $\sigma_{a_{m_{0}}}^{*}$ and $\lambda_{a_{m_{0}}}$ are positive constants. The same method can apply to $\sigma_{a_{m_{1}}}, \sigma_{b_{m_{0}}}$, and $\boldsymbol{\sigma}_{*}$. The proposed learning rate adaptation method can effectively restrain large learning rates.

In conclusion, the indirect adaptive control algorithm now proceeds as follows: first, adjust the adjustable parameters of the mathematical model using (13) and (21); next, update the parameters of the ideal model using (17). The proposed strategy can effectively avoid the nonconvergence problem of the adjustable parameters caused by measurement

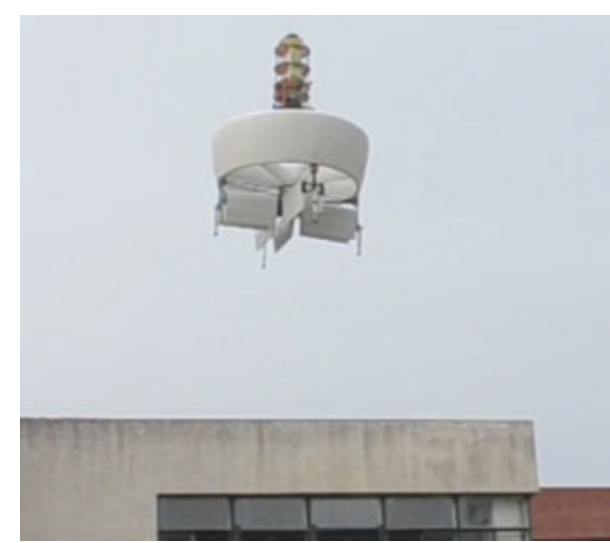

FIGURE 3: Flight test.

noises and can considerably reduce the tracking error during the adaptive learning process, especially in the initial stage.

\section{Test}

In this section the performance of the proposed indirect adaptive control strategy is demonstrated by a series of numerical simulations and flight tests, shown in Figure 3. For the ducted fan MAV, the indirect adaptive control software runs on a DSP, and the sensor units (including mechanical gyroscopes and angular rate gyroscopes) mounted on the top of the fuselage provide the measured data of the Ducted fan MAV, including the heading of the moving platform and three-axis angular rates. Of course, the sensors outputs contain measurement noises. follows:

All flight tests begin with the trim conditions given as

(1) ideal model: $a_{m_{0}}^{*}=9.0, a_{m_{1}}^{*}=4.2$, and $b_{m_{0}}^{*}=36.0$;

(2) error compensator: $k_{\theta_{e}}=3.6, k_{q_{e}}=1.7$;

(3) proportional and differential coefficients: $\tau_{\theta}=0.5$, $\tau_{q}=2.0$

(4) initial values: $a_{m_{0}}(0)=4.0, a_{m_{1}}(0)=2.0, b_{m_{0}}(0)=$ 10.0 , and $\mathbf{K}_{*}(0)=0$;

(5) learning rates: $\sigma_{a_{m_{0}}}^{*}=0.2, \sigma_{a_{m_{1}}}^{*}=0.2, \sigma_{b_{m_{0}}}^{*}=0.1$, $\sigma_{u}^{*}, \sigma_{w}^{*}, \sigma_{p}^{*}, \sigma_{\delta_{r}}^{*}, \sigma_{\delta_{t}}^{*}, \sigma_{\eta}^{*}=0.1$, and $\lambda_{\times}=1.5$.

Three different tests have been conducted to demonstrate the advantages of the indirect adaptive controller. The performance of the proposed controller is also compared with other existing controllers designed with the same assumptions.

4.1. Numerical Simulation. The numerical simulation uses a model of the MAV, as proposed by Ning [29], with adding noises to the model outputs. The parameters of the model are identified on the basis of a set of flight data. The responses versus time are shown from 0 to $600 \mathrm{~s}$ with adaption stopped at $180 \mathrm{~s}$, shown in Figure 4. The tracking error is very small even in the initial stage of the adaptive 


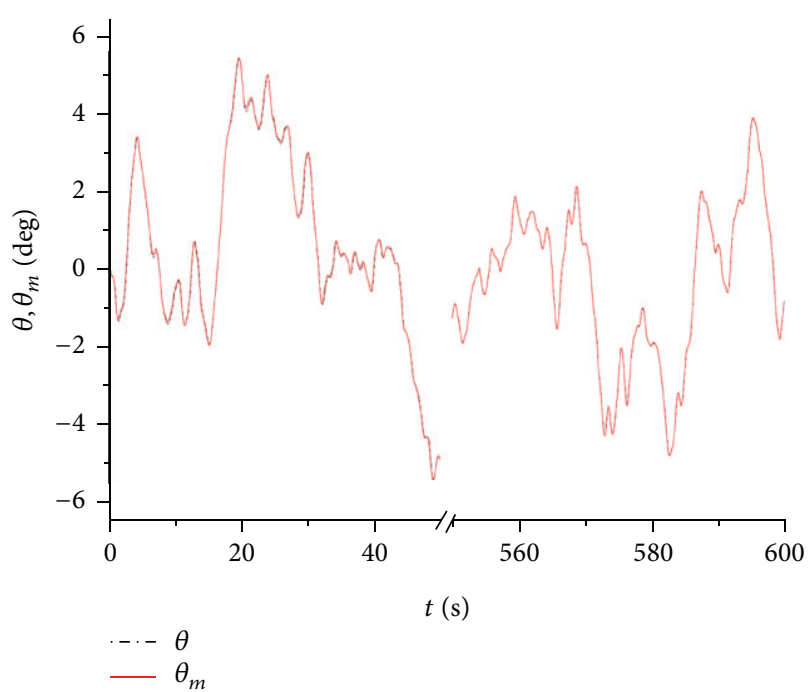

(a) Pitch angle

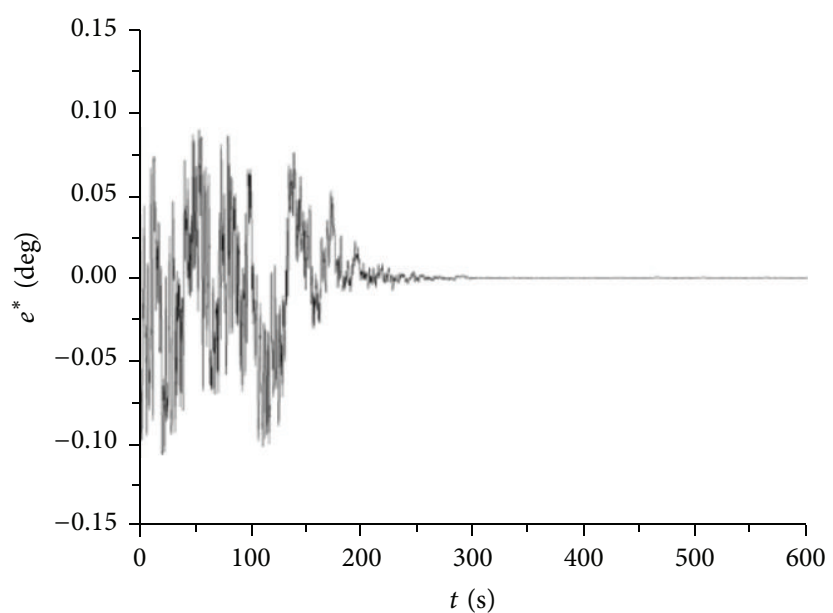

(c) The noise-free tracking error $\left(e^{*}\right)$

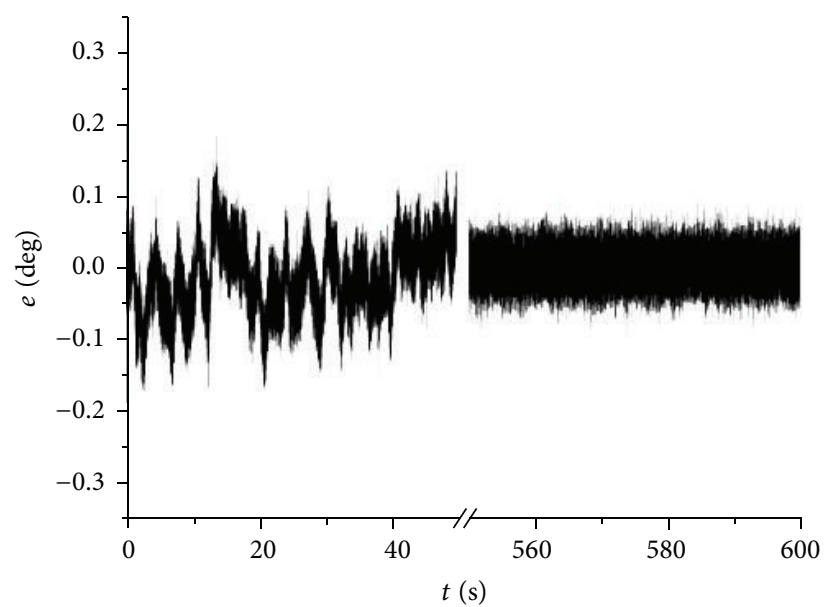

(b) Tracking error with noise

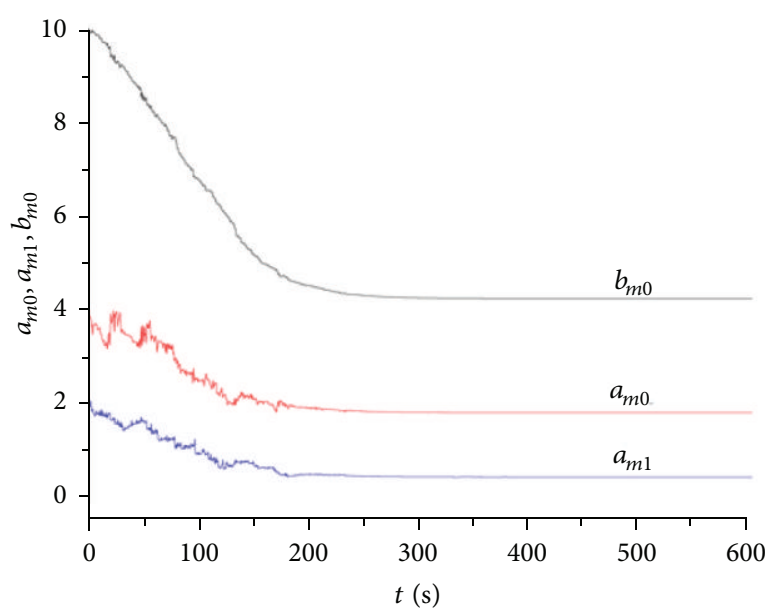

(d) Parameter learning process for $a_{m_{0}}, a_{m_{1}}, b_{m_{0}}$

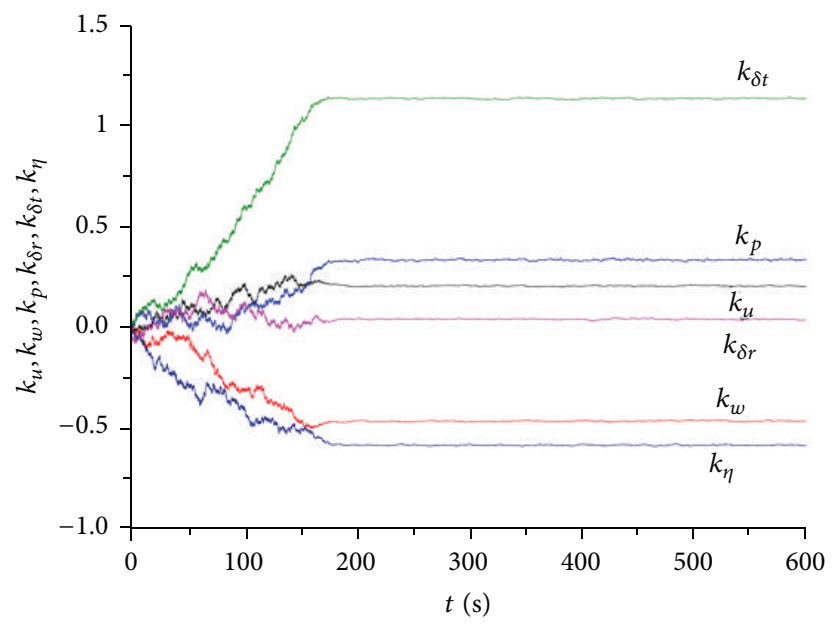

(e) Parameter learning process for $\mathbf{K}_{*}$

Figure 4: Numerical simulation results. 


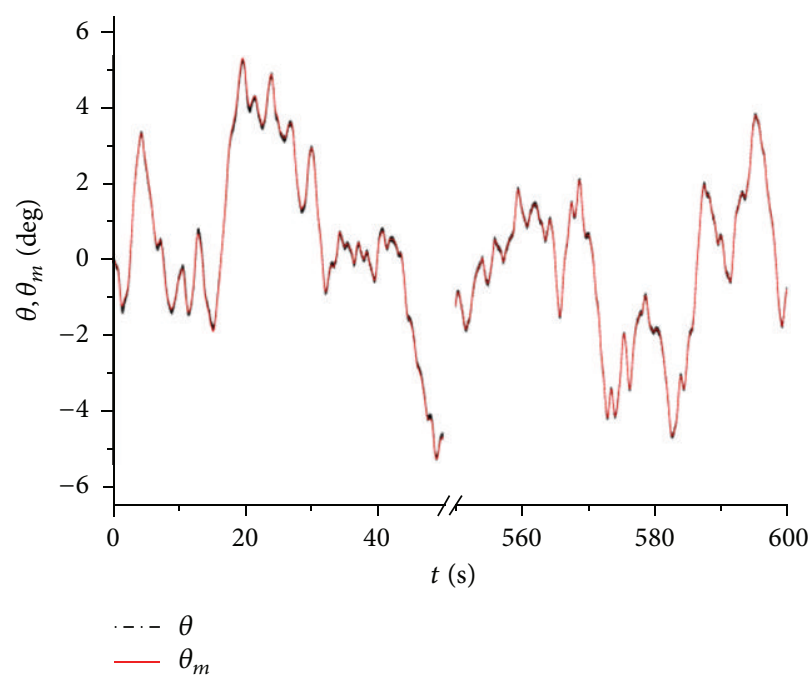

(a) Pitch angle

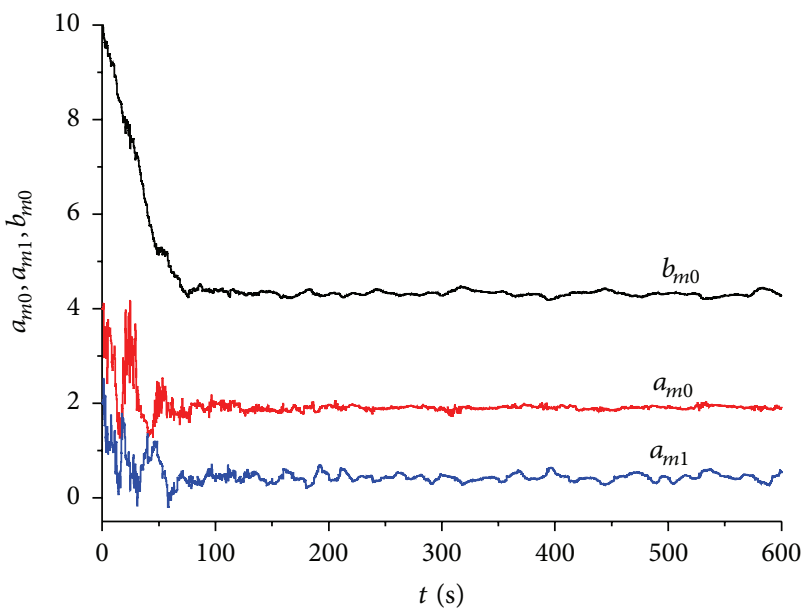

(c) Parameter learning process for $a_{m_{0}}, a_{m_{1}}, b_{m_{0}}$

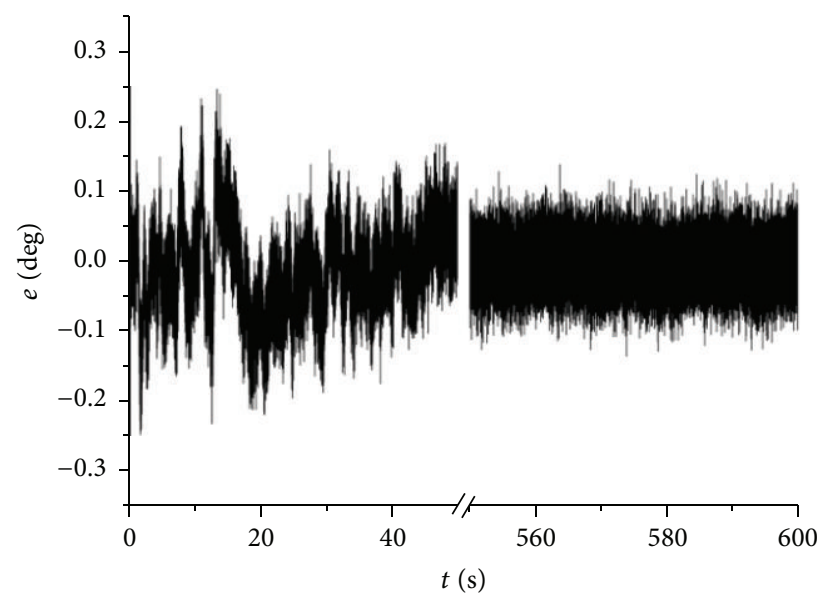

(b) Tracking error

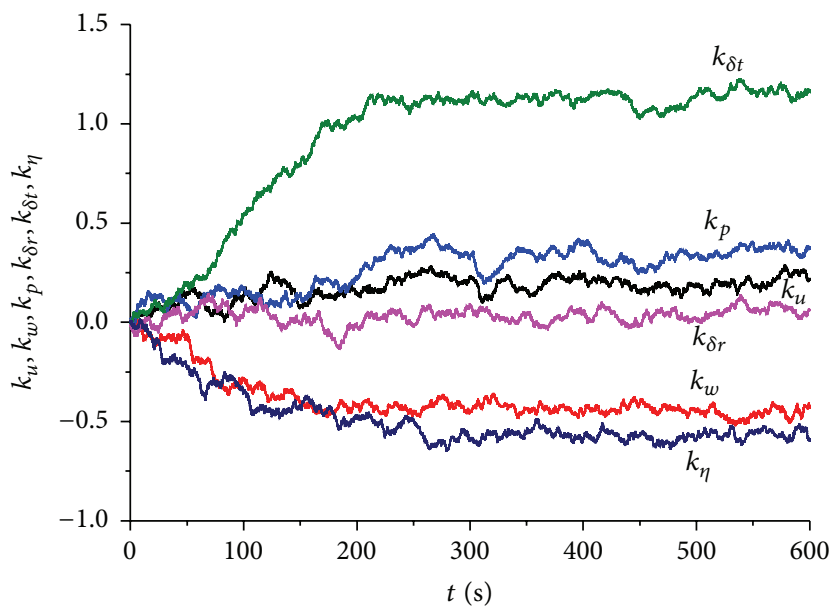

(d) Parameter learning process for $\mathbf{K}_{*}$

FIGURE 5: Flight test results with error compensation.

learning process. The learning process of adjustable parameters quickly reaches a near-steady state, which illustrates the convergence of adjustable parameters. The noise-free tracking error $e^{*}$ gradually reduces to zero, which explicitly shows that the proposed controller can achieve satisfactory tracking performance in the presence of measurement noises.

4.2. Flight Test. The proposed controller is now demonstrated by using the full MAV dynamics. The performances of the controller with error compensation are shown in Figure 5. The tracking error is reduced significantly in the initial stage of the adaptive learning process and is close to zero in the later stage. The learning process of the main adjustable parameters quickly reaches a near-steady state, which also illustrates the convergence of adjustable parameters with guaranteed tracking performance in the presence of measurement noises.

Compared with the above numerical simulation results, adjustable parameters exhibit considerable variation because the complete model with actuator dynamics and the saturation constraints on the inputs have been incorporated during the flight test. However, adjustable parameters converge fast because the significant tracking error can accelerate the adaptive learning rates. Note that the tracking performance and the convergence of adjustable parameters deteriorate if the learning rate adaptation method is not employed.

The experimental results without error compensation are shown in Figure 6. The tracking error increases considerably in the initial stage of the adaptive learning process. However, the convergence of adjustable parameters remains unchanged, and the learning process of main adjustable parameters extends over a slightly shorter period of time. The proposed learning rate adaptation strategy can also eliminate the adverse effect of large adaptive learning rates on the convergence of adjustable parameters.

4.3. Comparison between the Proposed and Existing Adaptive Controller. The experimental results of the existing adaptive 


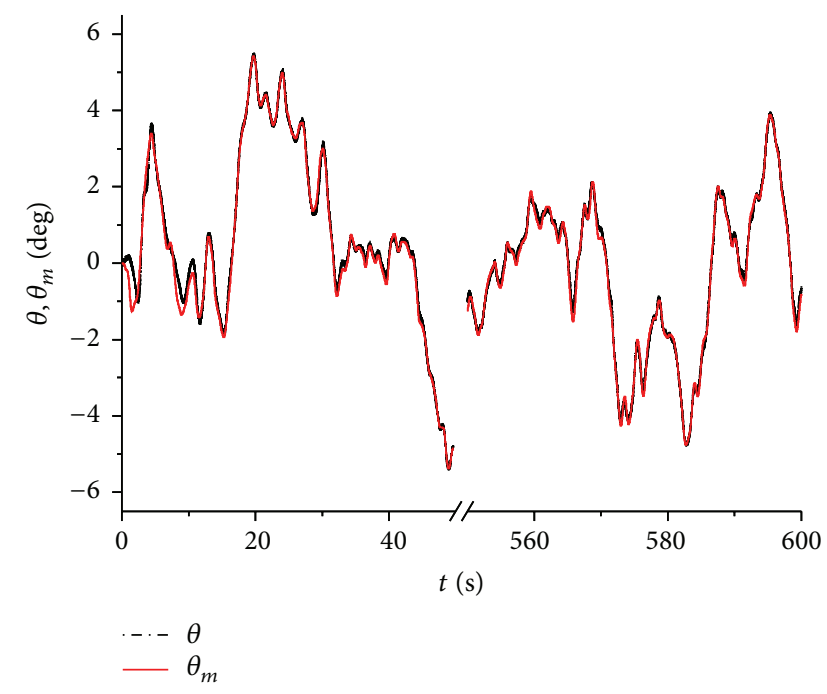

(a) Pitch angle

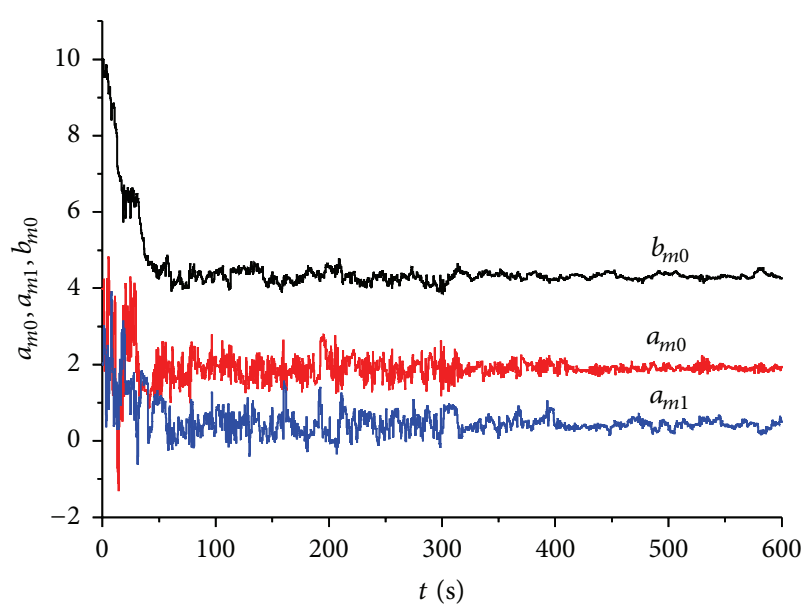

(c) Parameter learning process for $a_{m_{0}}, a_{m_{1}}, b_{m_{0}}$

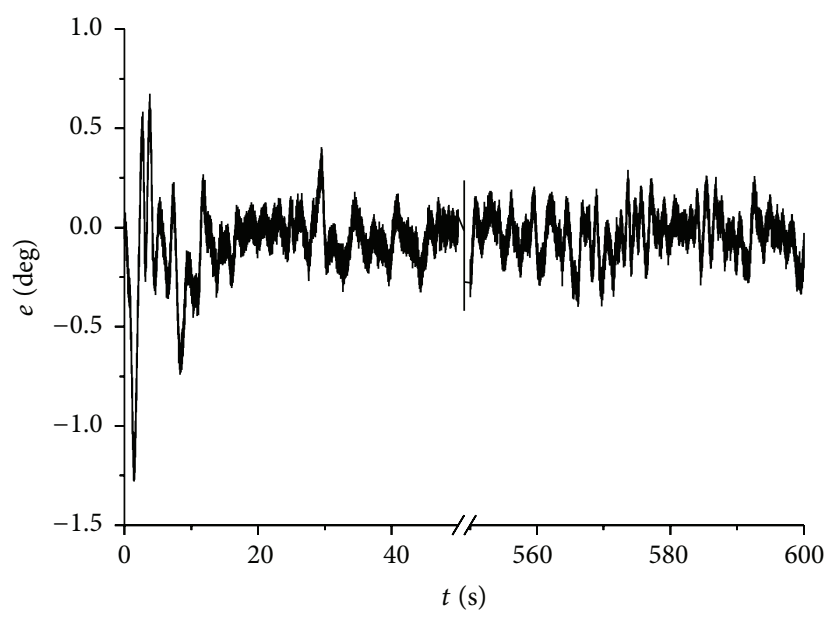

(b) Tracking error

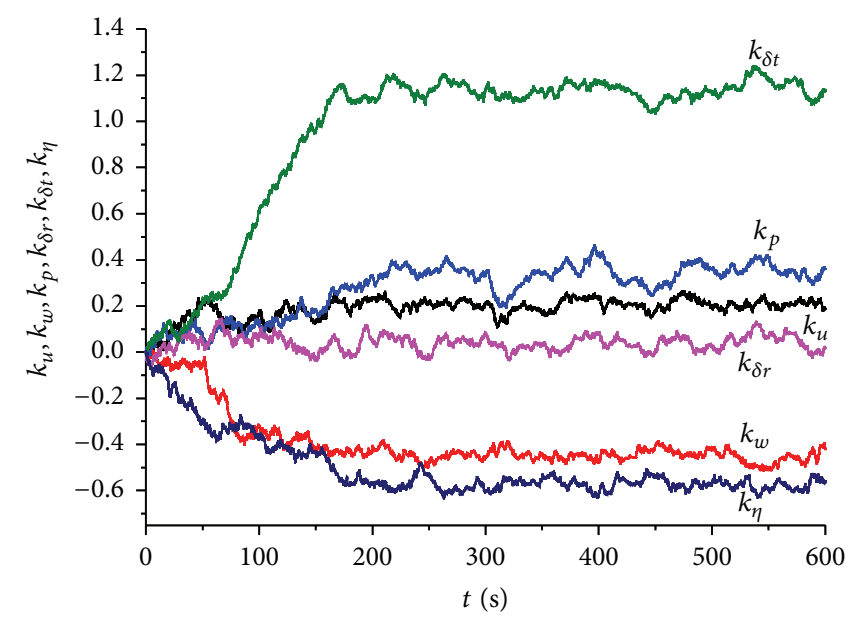

(d) Parameter learning process for $\mathbf{K}_{*}$

FIGURE 6: Flight test results without error compensation.

controller without any noise reduction strategy in [27], as shown in Figure 7, indicate that the tracking performance deteriorates significantly during the whole learning process. Meanwhile, $k_{c}, k_{q}, k_{\theta}$, and $\mathbf{K}_{*}$, the adjustable parameters, slowly diverge.

The results also demonstrate that the existing controller lacks robustness in the presence of measurement noises, though the adaptive algorithm uses a combination of lowand high-pass filters.

4.4. Test Summary. The experimental results show that the indirect adaptive controller effectively solves the unbiased and convergent problem of adjustable parameters caused by the measurement noises and therefore achieves satisfactory tracking performance. Figure 4 shows that the proposed controller exhibits a perfect learning behavior and eliminates the steady-state bias. By comparing the results illustrated in Figures 5, 6, and 7, it can be concluded that the error compensation strategy can noticeably reduce the tracking error, especially in the initial stage of the adaptive learning process, and the proposed controller outperforms the existing adaptive controllers in terms of the tracking error and the convergence of adjustable parameters.

\section{Conclusions}

This paper presents the detailed design of the indirect adaptive attitude tracking controller for the ducted fan MAV. The proposed indirect adaptive controller is stable and robust and shows significant improvement in performance over the existing adaptive controllers in the presence of measurement noises. It is able to effectively reduce the tracking error in the initial stage of the adaptive learning process because of the error compensation strategy and overcome the effect of measurement noises on the convergence of adjustable parameters. Moreover, the learning rate adaptation strategy can further minimize the effect of large adaptive learning 


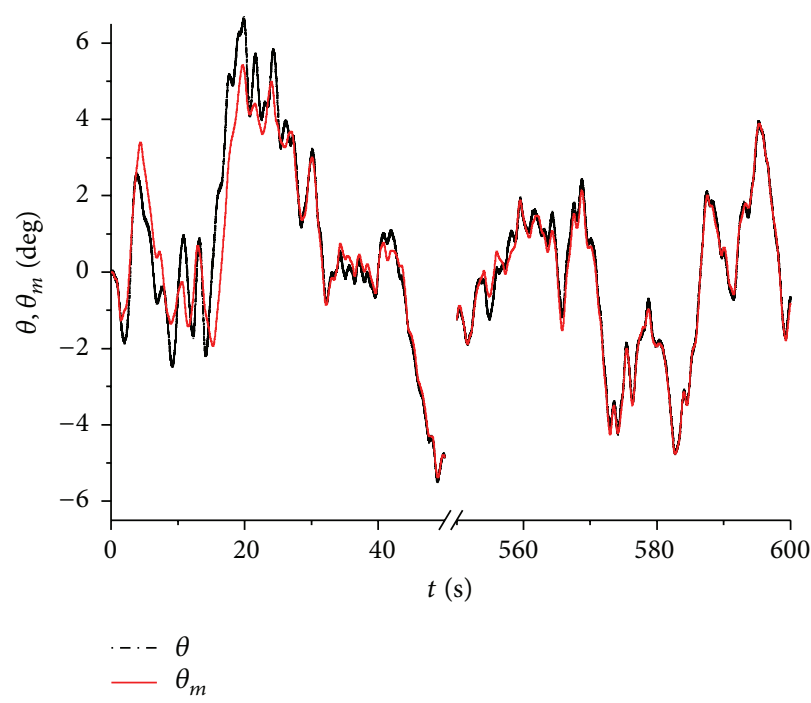

(a) Pitch angle

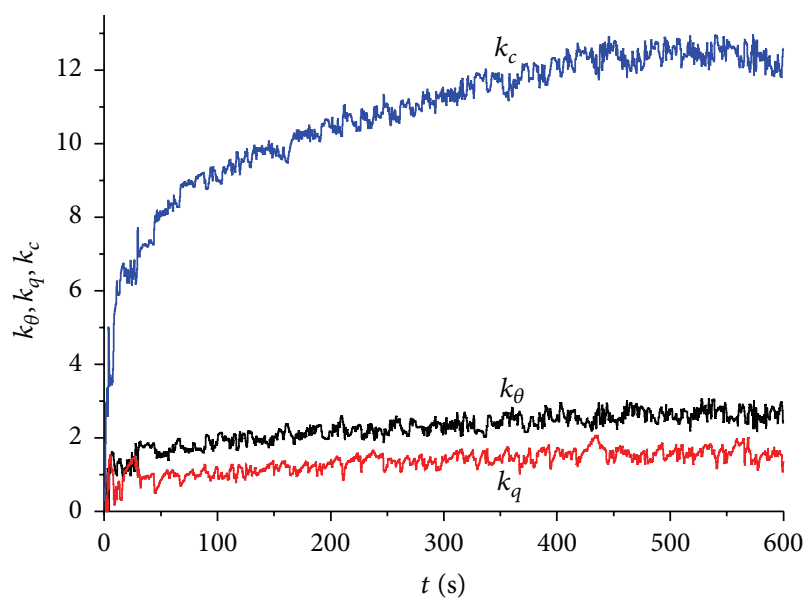

(c) Parameter learning process for $k_{c_{m}}, k_{q_{m}}, k_{\theta_{m}}$

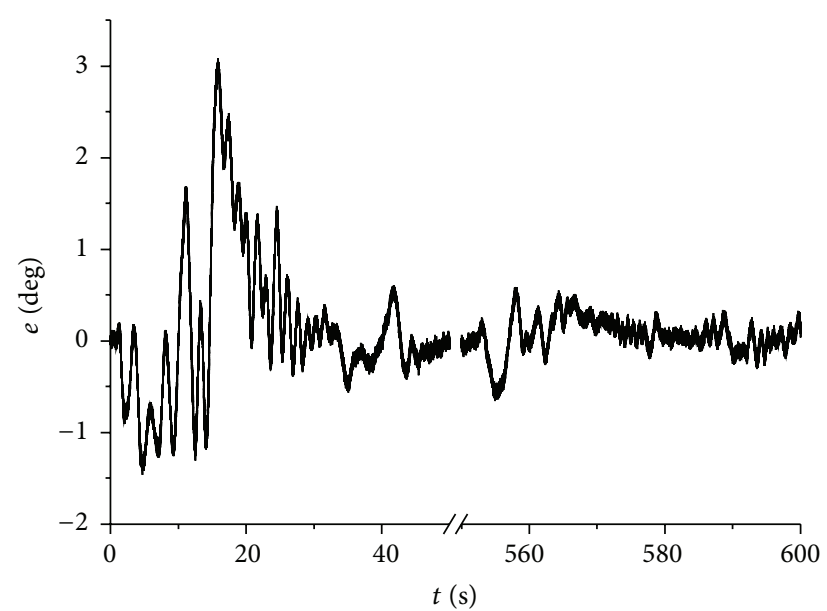

(b) Tracking error

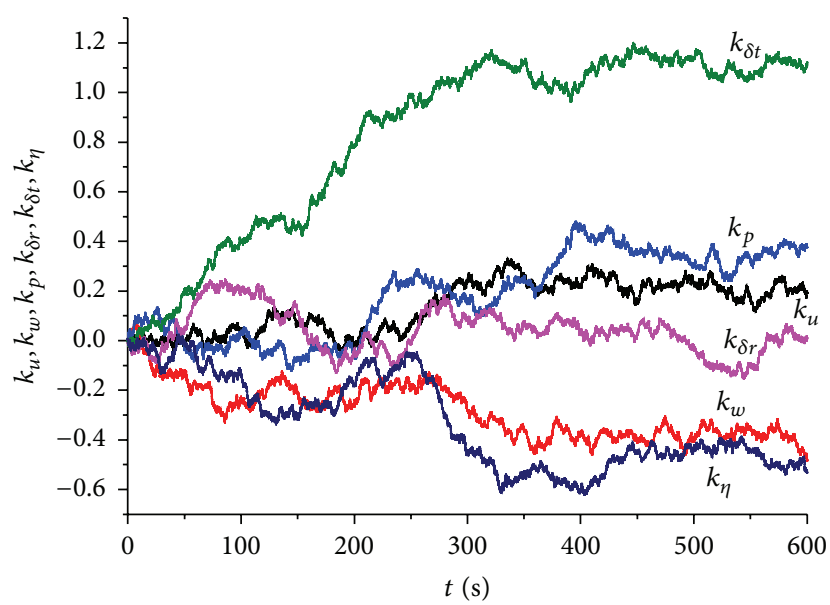

(d) Parameter learning process for $\mathbf{K}_{*}$

FIGURE 7: Flight test results of the existing adaptive controller in [27].

rates on the convergence of adjustable parameters. The experimental results have verified the proposed adaptive controller.

\section{Nomenclature}

Symbols

$\begin{array}{ll}\text { A: } & \text { System matrix } \\ a_{0}, a_{1}, b_{0}: & \text { Plant parameters } \\ a_{m_{0}}, a_{m_{1}}, b_{m_{0}}: & \text { Adjustable model parameters } \\ \mathbf{B}: & \text { Control matrix } \\ \widehat{D}: & \text { Characteristic polynomial of } e(s) \\ E: & \text { Expectation } \\ \mathbf{e}: & \text { Equivalent trim disturbance } \\ \mathbf{e}_{u}, \mathbf{e}_{x}: & \text { Estimate errors of trim input and trim } \\ e_{:} & \text {state vectors } \\ f: & \text { Tracking error } \\ \mathbf{K}_{*}: & \text { Nonlinear kinematic function }\end{array}$

$k_{c_{m}}, k_{q_{m}}, k_{\theta_{m}}:$ Adjustable parameters

$k_{q_{e}}, k_{\theta_{e}}: \quad$ Coefficients of error compensator

$M_{\bullet}^{\dot{q}}: \quad$ Aerodynamic parameter

$\mathbf{M}_{*}^{\dot{q}}: \quad \quad$ Aerodynamic parameter vector

$q, p, r: \quad$ Linearized roll, pitch, and yaw rates,

$\widehat{q}, \widehat{p}, \widehat{r}: \quad$ Roll, pitch, and yaw rates, deg/s

$\widehat{\mathbf{u}}, \mathbf{u}: \quad$ System input and its linearized version

$\mathbf{u}_{e}, \mathbf{u}_{0}: \quad$ Trim input and nominal trim input

$u, v, w$ : Linearized forward, lateral, and vertical velocities, $\mathrm{m} / \mathrm{s}$

$\widehat{u}, \widehat{v}, \widehat{w}: \quad$ Forward, lateral, and vertical velocities,

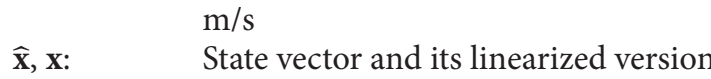

$\mathbf{x}_{e}, \mathbf{x}_{0}$ : Trim state and nominal trim state

$\Delta: \quad$ Increment or difference

$\tilde{\delta}_{*}: \quad$ Equivalent input disturbance

$\boldsymbol{\delta}_{*}: \quad$ Input disturbance vector

$\delta_{p}, \delta_{r}, \delta_{y}, \delta_{t}$ : Linearized pitch, roll, yaw, and throttle control, deg 


$\begin{array}{ll}\delta_{p a}: & \text { Plant input, deg } \\ \delta_{p b}: & \text { Adjustable system input, deg } \\ \delta_{p c}: & \text { Manipulated input, deg } \\ \widehat{\delta}_{p}, \widehat{\delta}_{r}, \widehat{\delta}_{y}, \widehat{\delta}_{t}: & \text { Pitch, roll, yaw, and throttle control, deg } \\ \varepsilon: & \text { Generalized error with noise } \\ \varepsilon_{0}: & \text { Noise-free generalized error } \\ \eta: & \text { Unknown input disturbance } \\ \lambda: & \text { Positive constant } \\ \Theta: & \text { Uncertain aerodynamic parameter set } \\ \theta, \phi, \psi: & \text { Linearized pitch, roll, and yaw angles, deg } \\ \theta_{0}: & \text { Unknown true value of pitch angle, deg } \\ \theta_{m}: & \text { Adjustable system output, deg } \\ \dot{\theta}, \dot{\theta}_{0}: & \text { Pitch derivative and its unknown true } \\ \hat{\theta}, \widehat{\phi}, \widehat{\psi}: & \text { value, deg/s } \\ \sigma, \sigma^{*}, \sigma_{*}: & \text { Pitch, roll, and yaw angles, deg } \\ \tau: & \text { Adaptive learning rate } \\ \xi: & \text { Differential or proportional coefficient } \\ & \text { Measurement noise. }\end{array}$

\section{Subscripts}

$\begin{array}{ll}a_{m_{0}}, a_{m_{1}}, b_{m_{0}}: \text { Adjustable model parameters } \\ p: & \text { Pitch } \\ q: & \text { Pitch rate } \\ r: & \text { Roll } \\ t: & \text { Throttle } \\ u: & \text { Forward velocity } \\ w: & \text { Vertical velocity } \\ y: & \text { Yaw } \\ \delta_{r}: & \text { Roll cyclic } \\ \delta_{t}: & \text { Main rotor collective } \\ \varepsilon: & \text { Generalized error } \\ \eta: & \text { Input disturbance } \\ \theta: & \text { Pitch angle } \\ \bullet: & u, v, w, \theta, \phi, \psi, q, p, r, \delta_{p}, \delta_{r}, \delta_{y}, \text { and } \delta_{t} \\ \times: & a_{m_{0}}, a_{m_{1}}, b_{m_{0}}, u, w, p, \delta_{r}, \text { and } \delta_{t} .\end{array}$

\section{Conflict of Interests}

The authors declare that there is no conflict of interests regarding the publication of this paper.

\section{Acknowledgments}

This study was supported by the Fundamental Research Funds for the Central Universities under Grant no. NS2013032. The authors would like to thank two anonymous reviewers for helpful comments.

\section{References}

[1] US Department of Defense, UAV Road Map, US Department of Defense, Washington, DC, USA, 2003.

[2] D. C. Dugan, "Trust control of VTOL aircraft part deux," in Proceedings of the 5th Decennial AHS Aeromechanics Specialists' Conference, American Helicopter Society, San Francisco, Calif, USA, January 2014.
[3] J.-M. Pflimlin, P. Binetti, P. Souères, T. Hamel, and D. Trouchet, "Modeling and attitude control analysis of a ducted-fan micro aerial vehicle," Control Engineering Practice, vol. 18, no. 3, pp. 209-218, 2010.

[4] W. L. Cook, "Summary of lift and lift/cruise fan powered lift concept technology," NASA Technical Report, NASA's Ames Research Center, Moffett Field, Calif, USA, 1993.

[5] J. P. Cycon, "Decoding the cypher UAV," Vertiflite, vol. 36, no. 6, pp. 56-58, 1990.

[6] D. Walsh and J. P. Cycon, "The Sikorsky Cypher UAV: a multipurpose platform with demonstrated mission flexibility," in Proceedings of the 54th American Helicopter Society Annual Forum, vol. 2, pp. 1410-1418, Washington, DC, USA, May 1998.

[7] L. Lipera, J. Colbourne, M. Tischler, M. Mansur, M. Rotkowitz, and P. Patangui, "The micro craft istar micro-air vehicle: control system design and testing," in Proceedings of the 57th Annual Forum of the American Helicopter Society, pp. 1-11, Washington, DC, USA, 2001.

[8] J. M. Pflimlin, P. Souères, and T. Hamel, "Hovering flight stabilization in wind gusts for a ducted fan UAV," in Proceedings of the 43rd IEEE Conference on Decision and Control (CDC'04), pp. 3491-3496, Paradise Island, Bahamas, December 2004.

[9] P. Martin and D. Boxwell, "Design, analysis and experiments on a 10-inch ducted rotor VTOL UAV," in Proceedings of the AHS International Specialists' Meeting on Unmanned Rotorcraft, American Helicopter Society, San Marcos, Calif, USA, 2005.

[10] J. Pereira, I. Chopra, and A. Gessow, "Effects of shroud design variables on hover performance of a shrouded rotor for micro air vehicle applications," in Proceedings of the AHS International Specialists' Meeting on Unmanned Rotorcraft, American Helicopter Society, San Marcos, Calif, USA, 2005.

[11] D. N. Salluce, Comprehensive system identification of Ducted fan UAVs [Ph.D. thesis], California Polytechnic State University, San Luis Obispo, Calif, USA, 2004.

[12] E. N. Johnson and M. A. Turbe, "Modeling, control, and flight testing of a small ducted fan aircraft," Journal of Guidance, Control, and Dynamics, vol. 29, no. 4, pp. 769-779, 2006.

[13] A. Ko, O. J. Ohanian, and P. Gelhausen, "Ducted Fan UAV modeling and simulation in preliminary design," in Proceedings of the AIAA Modeling and Simulation Technologies Conference, pp. 161-180, August 2007.

[14] N. Metni, J.-M. Pflimlin, T. Hamel, and P. Souères, "Attitude and gyro bias estimation for a VTOL UAV," Control Engineering Practice, vol. 14, no. 12, pp. 1511-1520, 2006.

[15] I. K. Peddle, T. Jones, and J. Treurnicht, "Practical near hover flight control of a ducted fan (SLADe)," Control Engineering Practice, vol. 17, no. 1, pp. 48-58, 2009.

[16] S. Sheng, A. A. Mian, Z. Chao, and B. Jiang, "Autonomous takeoff and landing control for a prototype unmanned helicopter," Control Engineering Practice, vol. 18, no. 9, pp. 1053-1059, 2010.

[17] R. Naldi, L. Gentili, L. Marconi, and A. Sala, "Design and experimental validation of a nonlinear control law for a ductedfan miniature aerial vehicle," Control Engineering Practice, vol. 18, no. 7, pp. 747-760, 2010.

[18] A. Manouchehri, H. Hajkarami, and M. S. Ahmadi, "Hovering control of a ducted fan VTOL Unmanned Aerial Vehicle (UAV) based on PID control," in Proceedings of the 2nd Annual Conference on Electrical and Control Engineering (ICECE '11), pp. 5962-5965, IEEE, Yichang, China, September 2011.

[19] R. A. Hess and T. M. Ussery, "Sliding mode techniques applied to the control of a micro-air vehicle," in Proceedings of the AIAA 
Guidance, Navigation, and Control Conference, pp. 11-14, AIAA, 2003.

[20] C. M. Spaulding, M. H. Mansur, M. B. Tischler, R. A. Hess, and J. A. Franklin, "Nonlinear inversion control for a ducted fan UAV," in Proceedings of the AIAA Atmospheric Flight Mechanics Conference and Exhibit, AIAA 2005-6231, San Francisco, Calif, USA, 2005.

[21] F. L. Bras, R. Mahony, T. Hamel, and P. Binetti, "Adaptive filtering and image based visual servo control of a ducted fan flying robot," in Proceedings of the 45th IEEE Conference on Decision and Control (CDC '06), pp. 1751-1757, December 2006.

[22] H. B. Duan and C. H. Sun, "Pendulum-like oscillation controller for micro aerial vehicle with ducted fan based on LQR and PSO," Science China Technological Sciences, vol. 56, no. 2, pp. 423-429, 2013.

[23] G. Avanzini, U. Ciniglio, and G. De Matteis, "Full-envelope robust control of a shrouded-fan unmanned vehicle," Journal of Guidance, Control, and Dynamics, vol. 29, no. 2, pp. 435-443, 2006.

[24] R. Aruneshwaran, S. Suresh, J. Wang, and T. K. Venugopalan, "Neural adaptive flight controller for ducted-fan UAV performing nonlinear maneuver," in Proceedings of the IEEE Symposium on Computational Intelligence for Security and Defense Applications (CISDA '13), pp. 51-56, IEEE, Singapore, April 2013.

[25] W. Lee and H. Bang, "Control of ducted Fan UAV by fuzzy gain scheduler," in Proceedings of the International Conference on Control, Automation and Systems (ICCAS '07), pp. 812-816, IEEE, October 2007.

[26] Z. Omar, Intelligent control of a ducted-fan VTOL UAV with conventional control surfaces [Ph.D. thesis], RMIT University, Melbourne, Australia, 2010.

[27] S. A. Neild, L. Yang, and D. J. Wagg, "A modified model reference adaptive control approach for systems with noise or unmodelled dynamics," Proceedings of the Institution of Mechanical Engineers, Part I: Journal of Systems and Control Engineering, vol. 222, no. 3, pp. 197-208, 2008.

[28] K. J. Åström and B. Wittenmark, Adaptive Control, Courier Corporation, 2013.

[29] Z. Ning, Ducted fan unmanned aircraft flight dynamics analysis and control study [M.S. thesis], Nanjing University of Aeronautics and Astronautics, Nanjing, China, 2011. 


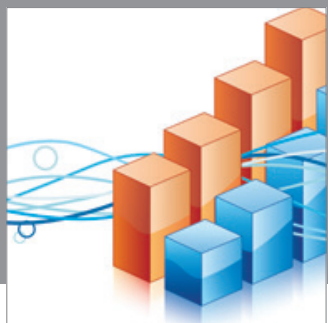

Advances in

Operations Research

mansans

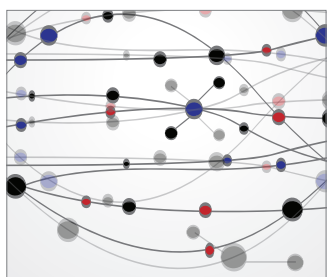

The Scientific World Journal
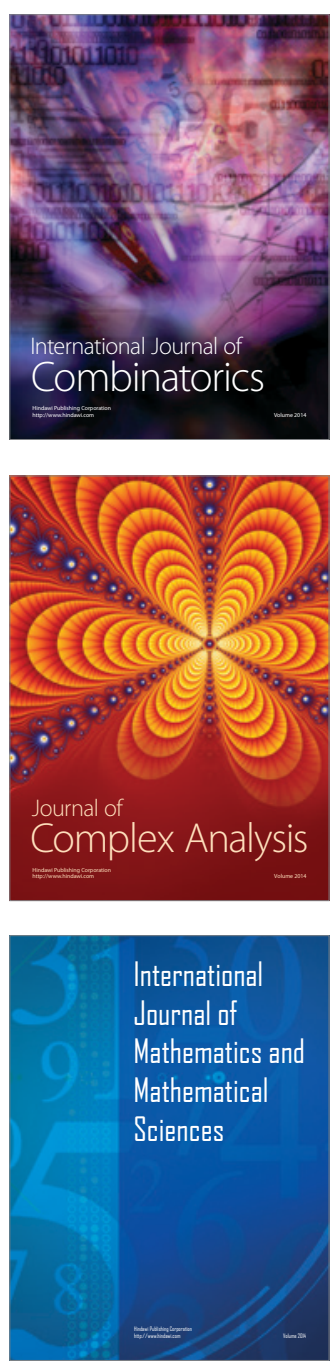
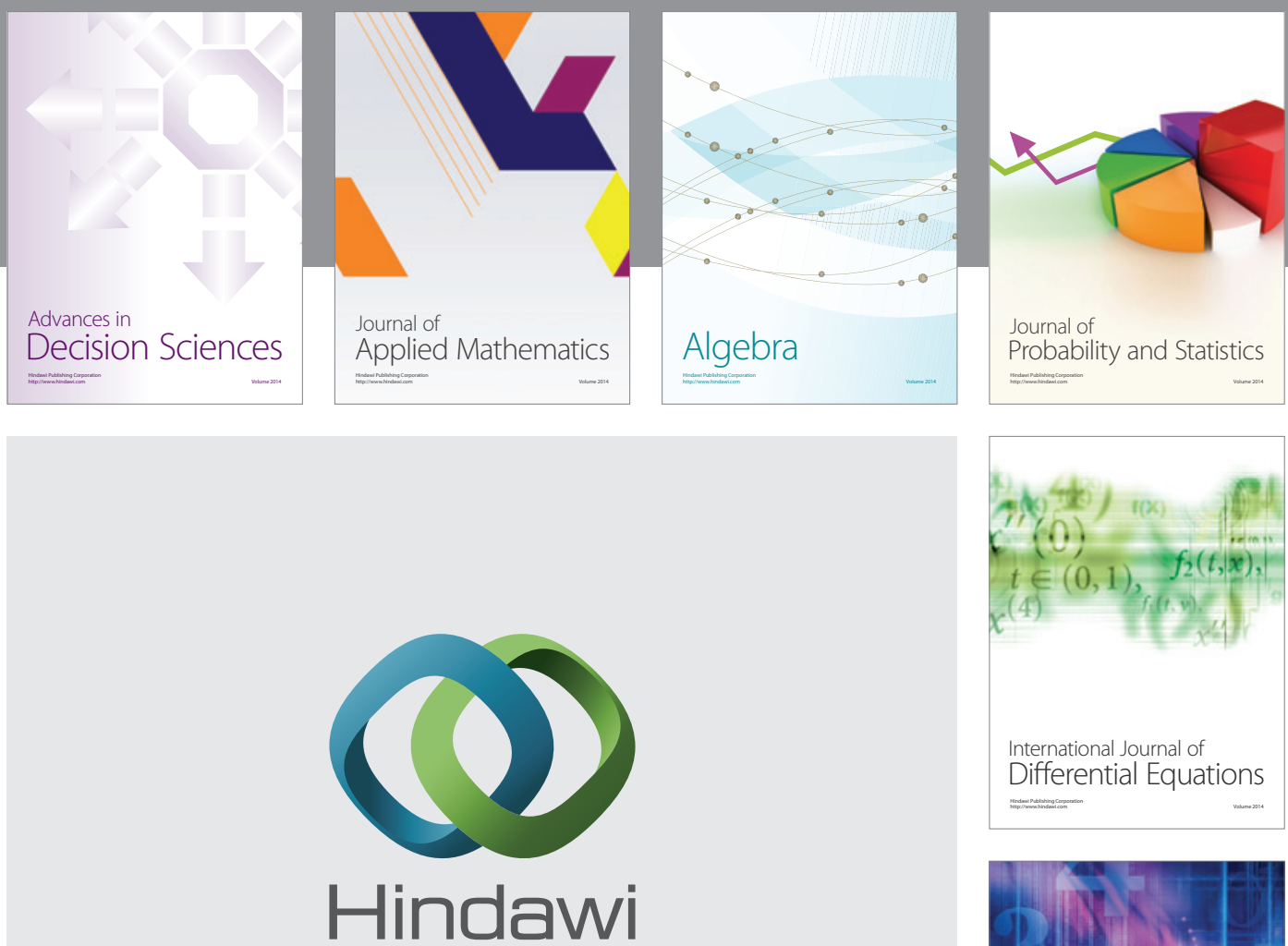

Submit your manuscripts at http://www.hindawi.com
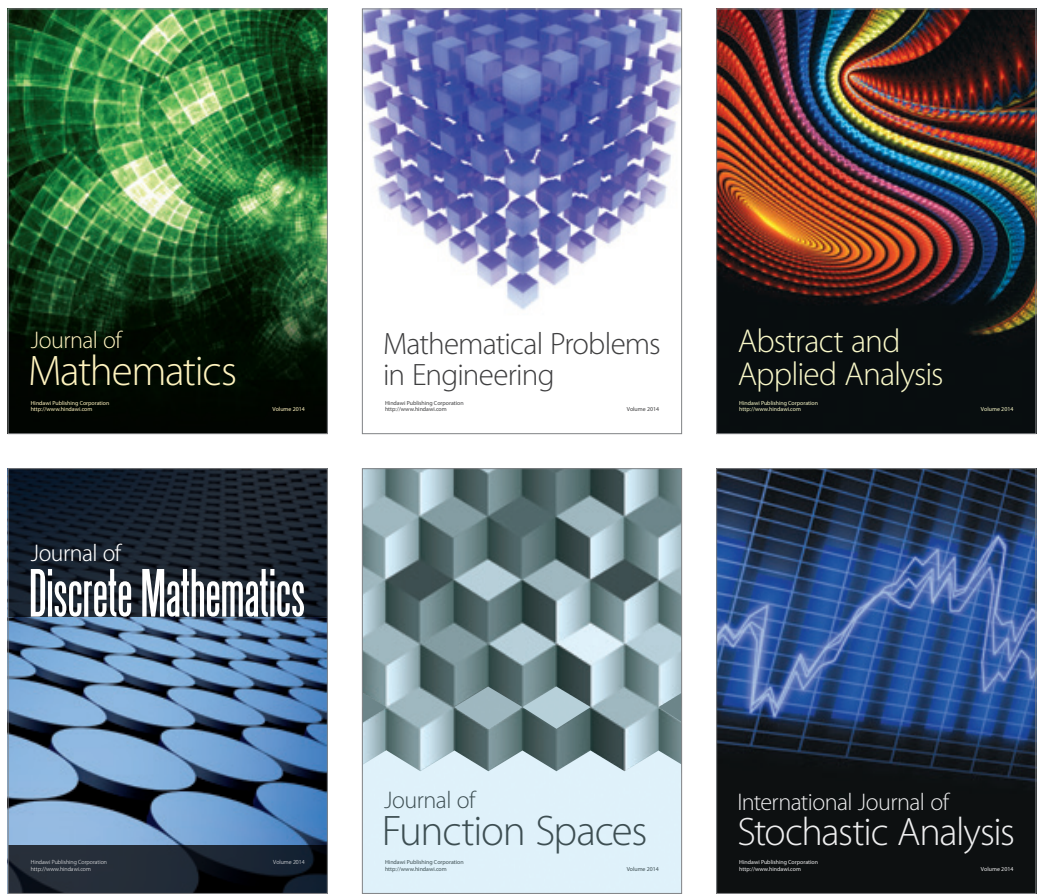

Journal of

Function Spaces

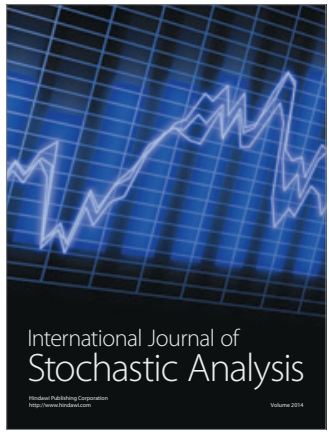

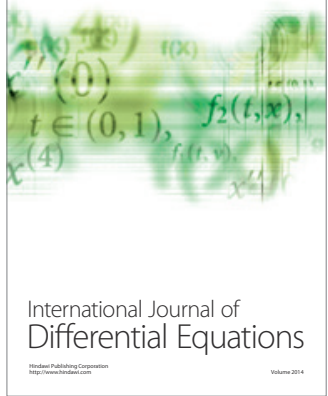
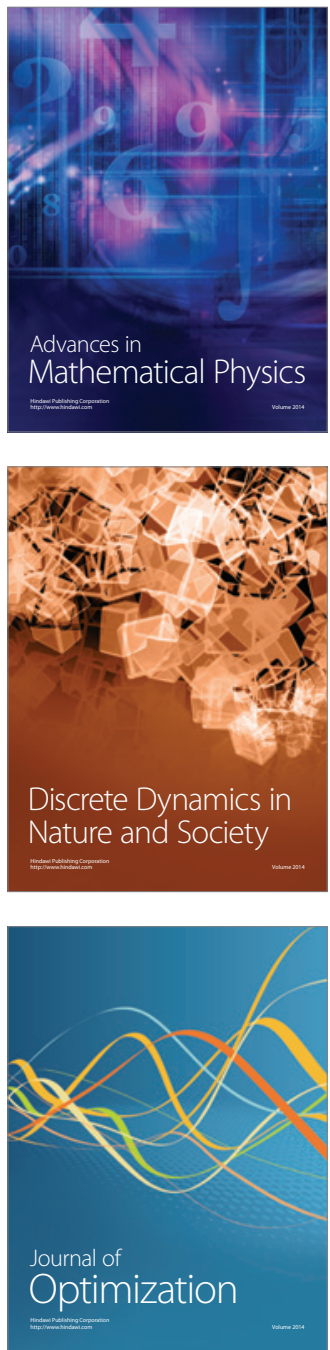